\title{
ITALIANISMI APPARTENENTI ALLA LINGUA PARLATA QUOTIDIANA NEGLI SCRITTI DI PETAR I E PETAR II PETROVIĆ NJEgoš
}

Cvijeta Brajičić, Università del Montenegro, cvijetab@ucg.ac.me

10.31902/fll.30.2020.10

UDK811.131.1'373.45PETROVIĆ NJEGOŠ P.II

Il tema di questo contributo è la presenza di italianismi appartenenti alla lingua parlata quotidiana negli scritti di vescovi e scrittori montenegrini, Petar I e Petar II Petrović Njegoš. Se si prendono in considerazione le affermazioni di esperti, secondo cui i prestiti provenienti dall'italiano e dai suoi dialetti rappresentano una parte integrante delle parlate popolari montenegrine della loro epoca, e il fatto che entrambi gli autori scelti hanno avuto l'opportunità di conoscere in vari modi la lingua italiana, sembra ragionevole presumere che il loro patrimonio scritto debba contenere elementi del lessico italiano. Questo argomento è stato scelto anche perché, esaminando la letteratura esistente dedicata ai lavori di Petar I e Petar II Petrović Njegoš, si è giunti alla conclusione che finora nessuno ha approfonditamente affrontato la presenza della componente lessicale italiana nei loro scritti, anche se ci sono accenni sporadici all'esistenza di materiale lessicale di origine italiana e veneziana nel linguaggio dei due autori citati sopra.

Parole chiave: Petar I Petrović, Petar II Petrović Njegoš, italianismi, parlate popolari, dialetto veneziano

\section{Introduzione}

Questo contributo tratta della presenza delle parole provenienti dall'italiano e dai suoi dialetti negli scritti dei vescovi e scrittori montenegrini, Petar I Petrović (1748-1830) e Petar II Petrović Njegoš (1813-1851). L'eredità scritta dei due vladika ${ }^{1}$ è stata oggetto di interesse da parte di molti esperti negli ultimi decenni. Le loro opere sono state studiate da diversi punti di vista; è stata analizzata anche la presenza di prestiti nella loro lingua, ma relativamente poca attenzione è stata dedicata alla componente lessicale italiana. Alcuni esperti

\footnotetext{
${ }^{1}$ Il titolo di vladika era proprio di principi vescovi montenegrini.
} 
linguisti, nei loro studi, hanno accennato alla presenza di italianismi negli scritti di Petar I e Petar II, ma un'analisi approfondita e argomentata è mancata. Paradossalmente, molta più attenzione è stata posta alla domanda se questi due autori conoscessero la lingua italiana. Nel caso di Petar I, gli studiosi sono giunti a conclusioni diametralmente opposte - alcuni sono convinti che il vescovo montenegrino, in una certa misura, conosceva l'italiano, mentre altri credono che egli non avesse nessuna possibilità di imparare questa lingua e che gli italianismi presenti nei suoi scritti provengano dalle parlate popolari, delle quali facevano parte integrante. La seconda ipotesi è sostenuta da Branislav Ostojić $(1976,12)$ che ha studiato le caratteristiche della lingua adoperata da Petar I nelle sue opere. Queste sue asserzioni sono in contrasto con quanto stabilito da Dušan Vuksan, il quale ha scoperto, nella biblioteca personale di Petar II Petrović Njegoš, alcuni titoli che possono essere attribuiti con certezza al suo predecessore. Anche Nikola Banašević $(1929,194)$ ritiene che Petar I conoscesse la lingua italiana, e un parere molto simile lo esprime pure Vesna Kilibarda $(2013,200)$. Questa autrice ha esaminato anche la conoscenza della lingua italiana di Petar II Petrović. Lei sostiene che l'italiano è stato la "prima lingua straniera che Njegoš, da giovane, ha avuto l'opportunità di imparare dal suo maestro, Josip Tropović (1775-1828), in una piccola scuola privata aperta nel 1812 presso una chiesa vicino a Castelnuovo" (Kilibarda 2013, 198). Facendo riferimento allo studio di Savo Vukmanović, pubblicato nella rivista montenegrina Boka, Kilibarda afferma che Njegoš avrebbe potuto imparare la lingua italiana nella stessa città anche dal frate cattolico Piero Prezidente (Vukmanović 1982, 338). A suo parere, Njegoš è stato incoraggiato all'apprendimento di questa lingua anche dallo zio, il vescovo Petar I, "perché anche lui ne era conoscitore", consapevole del fatto che lui e il suo successore avrebbero potuto trarne benefici nei costanti contatti con la popolazione e con le autorità degli ex possedimenti veneziani in Boka e in Dalmazia, in cui, anche sotto il dominio austriaco, l'italiano è rimasto lingua ufficiale dell'amministrazione locale (Kilibarda 2013, 199). Che Njegoš conoscesse questa lingua lo testimoniano anche $i$ volumi presenti nella sua biblioteca. Nell'elenco redatto da Dušan Vuksan sono stati registrati nove titoli italiani "che Njegoš ha ereditato dalla biblioteca dello zio, nonché dieci libri che egli stesso ha acquisito, insieme a diversi giornali d'epoca e riviste in italiano alle quali presumibilmente era abbonato" (Kilibarda 2014, 37). Njegoš ha avuto molte opportunità di migliorare la propria conoscenza dell'italiano a diretto contatto con persone madrelingua, visto che ha visitato il territorio dell'odierna Italia più volte e in diverse occasioni (Kilibarda 
2011, 289-302). Anche Svetozar Stijović (1992, 30) nel suo studio sostiene che Njegoš conosceva la lingua italiana citando, a sostegno di questa tesi, diverse testimonianze di alcuni contemporanei del vescovo.

\section{La lingua di Petar I Petrović}

Come già detto, la conoscenza della lingua italiana di Petar I e Petar II ha finora destato più interesse degli esperti rispetto alla presenza di italianismi nei loro scritti, e di conseguenza a questo argomento non è stata prestata sufficiente attenzione. Branislav Ostojić ha analizzato la presenza delle parole di origine italiana nelle opere di Petar I. Egli sostiene che il vescovo fin dall'inizio utilizza la parlata popolare con alcuni prestiti provenienti dalle recensioni russe e serbe dell'antico slavo ecclesiastico (Ostojić 1985, 29). Inoltre, Ostojić afferma che negli scritti di Petar I è possibile notare l'influenza della poesia epica popolare, nonché la spiccata presenza di forestierismi, prima di tutto prestiti provenienti dalla lingua russa, ma anche parole di origine turca o italiana (Ostojić 1976, 12). La presenza della componente lessicale straniera, ritiene questo studioso, non è condizionata dalla conoscenza delle lingue straniere, ma in larga misura rappresenta la conseguenza di influssi diretti delle parlate popolari, le quali avevano già acquisito un numero piuttosto elevato di parole straniere. Ostojić è convinto che le scelte linguistiche di Petar I sono condizionate dagli argomenti che tratta, nonché dal pubblico a cui si rivolge. Nella sua corrispondenza ufficiale è dominante la componente di antico slavo ecclesiastico, mentre tali elementi sono quasi assenti nelle lettere private e nelle epistole. In queste ultime, a giudizio di Ostojić, Petar I variava la propria lingua in dipendenza dei destinatari - nelle epistole inviate ai clan degli altopiani è notevole la presenza delle parole di origine turca, mentre quelle destinate ai clan costieri contengono più italianismi (Ostojić 1985, 28). La nostra ricerca ha prodotto risultati diversi, ma questo sarà discusso più avanti.

\section{La lingua di Petar II Petrović Njegoš}

Quando si parla della lingua di Petar II, si deve anzitutto ricordare che egli era un sostenitore delle riforme linguistiche promosse da Vuk Stefanović Karadžić (1787-1864) e che, anche il vladika, come il suo predecessore Petar I, nelle proprie opere, utilizzava la varietà del parlato, caratteristica della città di Cettigne e delle zone limitrofe. Mihailo Stevanović sostiene che nelle prime opere di Njegoš (Svobodijada, poesie, Luča mikrokozma) è notevole la presenza di prestiti provenienti dalla recensione russa dell'antico slavo ecclesiastico, ma che il suo influsso è limitato esclusivamente alla sfera 
del lessico (Stevanović 1951, 17-31). Pertanto, a suo avviso, questi testi possono essere definiti scritti adoperando la varietà del parlato locale, sebbene la maggior parte dei contemporanei di Njegoš utilizzasse ancora la recensione russa dello slavo ecclesiastico. I recenti lavori di Petar II, secondo Stevanović, hanno un carattere completamente nazionale. Egli conclude che Njegoš stava cercando di attribuire le caratteristiche del parlato anche agli elementi "non popolari" che usava sporadicamente. Stevanović sottolinea che sarebbe tuttavia sbagliato uguagliare la lingua di Njegoš alla lingua promossa da Vuk Stefanović Karadžić. Njegoš, a suo parere, usa il vernacolo del suo luogo natio Montenegro e le zone circostanti (Stevanović 1951, 17-31). Drago Ćupić $(1988,130)$ sostiene che questo vescovo montenegrino nelle sue opere, oltre alla lingua nazionale, ha usato anche tanti forestierismi "soprattutto quelli provenienti dal greco, francese e italiano, e naturalmente - dalla lingua turca". La presenza di parole straniere in opere letterarie di Njegoš è stata confermata anche da Danilo Vušović $(1930,1)$, autore dello studio Prilozi proučavanju Njegoševa jezika. La componente lessicale italiana è stata registrata anche nel dizionario che accompagna l'edizione speciale di opera omnia di Petar II, a cura di Mihailo Stevanović e Radosav Bošković, pubblicata nell'ambito delle celebrazioni del $150^{\circ}$ anniversario della sua nascita. Tuttavia, questo vocabolario non comprende tutti, ma soltanto alcuni italianismi registrati in opere letterarie, scelti dagli autori che hanno voluto soffermarsi esclusivamente su alcuni esempi considerati, in qualche modo, interessanti o forse sconosciuti al grande pubblico.

\section{Caratteristiche del parlato in Montenegro tra la fine del Settecento e la prima metà dell'Ottocento}

Dal momento che, come si è visto sopra, la maggior parte degli esperti sostiene che Petar I e Petar II nei loro scritti hanno utilizzato il parlato della loro terra, riteniamo che sarebbe opportuno esporre per sommi capi le sue caratteristiche. Branislav Ostojić (1985) afferma che la caratteristica più importante della lingua montenegrina fino ai primi decenni dell'Ottocento è la mancanza di una struttura grammaticale salda, ed è proprio questa peculiarità a renderla suscettibile di varie influenze che si rispecchiano nella lingua scritta dei due vescovi. Egli conclude che nel Montenegro la lingua conserva a lungo le caratteristiche presenti nelle epoche precedenti, in particolar modo nel Medioevo. Questo va attribuito alla situazione socio-economica che non ha permesso la differenziazione di classi privilegiate in alcun modo, tantomeno in sfera culturale. Pertanto, la letteratura scritta che in passato di solito era legata ai ceti privilegiati in questo paese per secoli 
non è esistita e, quando ha iniziato a svilupparsi, era inseparabile dalla tradizione letteraria orale e costantemente sottoposta ai suoi influssi. Uno dei principali problemi della società montenegrina dell'epoca era la frammentazione, nonché l'esistenza di un gran numero di piccole celle isolate all'interno di ciascuno dei clan. Ciò nonostante, i legami tra di loro rimanevano forti, come lo erano le relazioni tra il Montenegro e le regioni costiere, sebbene esse appartenessero a un altro paese. Questi legami, secondo Ostojić $(1976,12)$, hanno influenzato anche gli scambi nella sfera della lingua. Egli ritiene che i contatti commerciali con le città costiere, in particolare con Cattaro, hanno fatto sì che nel linguaggio dell'entroterra si infiltrassero numerose parole di origine romanza. Inoltre, le circostanze socio-economiche hanno influenzato le migrazioni della popolazione dalle aree continentali verso la costa, il che ha influito sui vernacoli delle regioni costiere, sebbene l'influenza in senso opposto fosse molto più spiccata.

\section{Corpus}

Se si prendono in considerazione le affermazioni di esperti che gli italianismi costituiscono una parte integrante del parlato nel Montenegro durante il regno di Petar I e Petar II, e il fatto che essi in modi diversi - direttamente o indirettamente, hanno avuto la possibilità di conoscere la lingua italiana, sembra giustificato supporre che gli elementi del lessico italiano debbano essere presenti nei loro scritti. Tale ipotesi è confermata dalla ricerca che abbiamo condotto sul corpus costituito da tutti gli scritti di questi due autori montenegrini. Nel caso di Petar I il corpus comprende le sue epistole, la corrispondenza ufficiale e le opere poetiche realizzate negli ultimi decenni del XVIII e nei primi decenni del XIX secolo. Abbiamo deciso di includere nel corpus tutte e 16 le poesie presenti nell'edizione di CID, Podgorica (2001), benché nel volume pubblicato all'interno della collana Crnogorska književnost od XII do XIX vijeka (1996) ce ne siano solo 15. Infatti, l'editore di quest'ultima pubblicazione, Čedo Vuković, ritiene che Petar I non possa essere l'autore della poesia intitolata IV crnogorska, date le sue caratteristiche stilistiche e linguistiche. Questa decisione ci sembra legittima dal momento che questa poesia è stata inserita in tutte le altre edizioni delle opere di Petar I che abbiamo consultato.

Oltre alle poesie, Petar I ha scritto la Storia del Montenegro, il primo codice penale montenegrino, nonché un considerevole numero di lettere ed epistole. L'oggetto della nostra analisi sono, in primo luogo, le epistole scritte tra il 1785 e il 1830, destinate ai clan montenegrini e a quelli della costa Adriatica, così come le lettere ad 
alcuni suoi eminenti contemporanei. Nel patrimonio scritto di Petar I gli studiosi hanno individuato circa 300 epistole, mentre nel nostro corpus ne rientrano 245 , pubblicate nella prima edizione curata da Dušan Vuksan, e altre 13 pubblicate nella rivista Zapisi. Va notato che il numero preciso di epistole è abbastanza difficile da determinare, dato che alcuni testi di Petar I in certe edizioni sono classificati come epistole, e in altre come semplici lettere. II corpus comprende anche una serie di altri documenti di Petar I raccolti e pubblicati da Jevto Milović nel 1988 e nel 1989. II manoscritto della Storia del Montenegro non è stato conservato, ma la sua prima edizione a stampa, a cura di segretario di Njegoš Dimitrije Milaković, è stata pubblicata nell'almanacco Grlica nel 1835. Branislav Ostojić $(1985,27)$ sostiene che il testo sia diverso da quello originale, il che si può dedurre dalle annotazioni che lo accompagnano. II testo Zakonik opšti crnogorski i brdski è il primo documento legale montenegrino promulgato dall'Assemblea Nazionale tenutasi nel monastero Stanjevići alla fine di ottobre del 1798 (Andrijašević 2009, 79). La versione originale di questo codice conteneva 16 articoli, poi il documento è stato parzialmente modificato nel 1803 (Andrijašević 2009, 79). II patrimonio scritto di Njegoš è molto più ricco, benché la sua attività di scrittore si sia svolta per un breve arco di tempo di soli vent'anni. Si suppone che Njegoš abbia finito la sua prima opera, Glas kamenštaka, nel 1833. A causa dei problemi relativi all'autorizzazione alla stampa che doveva essere effettuata a Vienna, il manoscritto non è stato pubblicato. In seguito, è stato ampliato e stampato a Zemun nel 1854 con il titolo Svobodijada. Nel 1834 Njegoš ha scritto una raccolta di poesie chiamata Pustinjak cetinjski, e l'anno seguente un'altra, con il titolo Lijek jarosti turske che conteneva soltanto 4 poesie. Le opere Ogledalo srpsko e Luča mikrokozma sono state pubblicate a Belgrado nel 1845. La sua opera più nota, il poema Gorski vijenac, è stata stampata nel mese di febbraio del 1847 a Vienna, la città in cui erano stati pubblicati anche due brevi poemi di Njegoš - Kula Aleksića e Čardak Đurišića. La sua ultima opera Lažni car Šćepan Mali è stata stampata a Zagabria nel 1851, poco prima della sua morte (Vušović 1930, 6). Oltre alle sue opere letterarie, anche le lettere di Njegoš sono oggetto del nostro interesse. Esse, circa 1700, pubblicate dalla casa editrice Prosveta di Belgrado tra il 1950 e il 1955, sono state scritte in diverse occasioni e indirizzate a diversi stimati contemporanei del vladika montenegrino. Riteniamo che sia indispensabile prestare attenzione anche a questo segmento del patrimonio scritto di Njegoš per poter stabilire se, e in quale misura, i suoi contatti diretti con l'Italia e la conoscenza dell'arte e della cultura 
italiane abbiano potuto influire sulla sua lingua negli scritti di carattere non strettamente letterario.

II materiale raccolto è stato confrontato con gli italianismi registrati da Srđan Musić (1972) e da Vesna Lipovac Radulović (1997, 2004). Questi due autori hanno analizzato la presenza di italianismi nelle parlate popolari delle Bocche di Cattaro e di Paštrovići sulla costa adriatica, territori che hanno avuto contatti più stretti e più duraturi con l'Italia odierna rispetto ad altre regioni del Montenegro. I lavori dei due studiosi citati sopra sono stati scelti come termine di paragone allo scopo di stabilire se, e in quale misura, gli italianismi registrati nel nostro corpus coincidano con quelli presenti nel parlato, sebbene non si tratti della parlata popolare della terra natale di Petar I e Petar II, e nonostante il fatto che la presenza di parole provenienti dall'italiano nel linguaggio popolare sia stata esaminata quasi 150 anni dopo la morte di quest'ultimo. Il significato originale delle parole italiane è stato verificato utilizzando dizionari dell'uso della lingua italiana (Zingarelli 2000), nonché il vocabolario del dialetto veneziano (Boerio 1856, 1998).

\section{Italianismi nella lingua di Petar I Petrović}

Nella parte del corpus che contiene gli scritti di Petar I, abbiamo registrato 176 parole provenienti dalla lingua italiana o dal dialetto veneziano - 139 sostantivi, 20 verbi, 16 aggettivi e 1 avverbio. La maggior parte di questi termini è stata registrata nelle Epistole e nelle lettere, mentre gli italianismi sono molto meno presenti nel resto del suo patrimonio scritto. Contrariamente a quanto affermato da Branislav Ostojić $(1976,24)$, in quasi tutte le epistole si riscontra un numero simile di parole di origine italiana, e conseguentemente non si potrebbe concludere che l'incidenza di esse sia condizionata dalla provenienza dei destinatari. Sostanzialmente si tratta di un numero esiguo di termini che si ripetono. Nelle poesie di Petar I sono state registrate solo tre parole di origine italiana - sostantivi špag (it. spacco - spaccatura, fenditura, netta lacerazione, strappo), princip (it. principe - successore legittimo di una monarchia, persona di grande prestigio) e lubarda (it. bombarda - antica bocca da fuoco). Nel testo del Zakonik sono presenti i termini merginaš - persona addetta alla determinazione di confini tra proprietà terriere (it. margine - confine, limite) e kapitul (it. capitolo - ciascuna delle parti in cui è diviso un libro, sezione suddivisa in più parti di un contratto, di un regolamento o di una convenzione). Nella Storia di Petar I abbiamo registrato il nome konteja (it. contea - contado), qui nel significato di grande proprietà. I vocaboli rimanenti sono registrati nelle lettere e nelle epistole. Di questi termini 
74 (42,04\%) compaiono anche nei vocabolari di Musić e della Radulović, 13 parole $(7,38 \%)$ sono state registrate solo da Musić, mentre 22 vocaboli $(12,5 \%)$ sono stati riportati soltanto dalla Radulović. I rimanenti 67 termini $(38,06 \%)$ non sono stati registrati né nel parlato delle Bocche di Cattaro, né in quello di Budua e Paštrovići. I vocaboli che non sono stati registrati né dalla Radulović né da Musić, in maggior parte appartengono alla terminologia militare o amministrativa. Alcuni di questi sono: aleatski (it. alleato) - unito da un patto di alleanza, bail (it. ven. bailo) - chi ricopriva la carica di ambasciatore o console nelle colonie venete o fiorentine nel Levante, bregadijer (it. brigadiere) comandante di brigata, cirkuljarni kapetan (it. capitano del circolo) amministratore distrettuale, duka (it. duca) - nobile che nella gerarchia araldica è inferiore al principe e superiore al marchese; ob. condottiero, comandante, fregada (it. fregata) - veloce nave da guerra, kompanija (it. compagnia) - qui reparto di truppe che concorre a formare un battaglione, kapitan (it. capitano) - capo di esercito; nel Medioevo governatore, comandante di una città, kapetanijat (it. capitanato) l'edificio in cui risiedeva il capitano, karavela (it. caravella) - tipo di veliero, kavalijer (it. cavaliere) - qui chi è insignito di un'onorificenza cavalleresca, mandat (it. mandato) - ordine di eseguire qualcosa, oficijal (it. ufficiale) - qui grado militare, retor (it. arh. rettore) - titolare delle supreme cariche politiche, amministrative o giudiziarie, tenente kolonelo (it. tenente colonnello) - ufficiale di grado immediatamente inferiore al colonnello. Petar I utilizza anche alcuni toponimi italiani non presenti in vernacolo: Boka di Kataro (it. Bocche di Cattaro), Kastel Novi (it. Castel Nuovo) - Herceg Novi, Ragusa (it. Ragusa) - vecchio nome della città di Dubrovnik, Trieste (it. Trieste). I termini non registrati né da Musić né dalla Radulović, ma attestati nel corpus sono i seguenti: okazija (it. occasione) - possibilità, parta (it. parte) - sezione, porzione; fazione, partito, rebel (it. ribelle) - insorto, rivoltoso, stiman (it. stimato) - apprezzato, sudit (it. suddito) - il soggetto che si trova in una condizione di dipendenza dalla sovranità dello stato. I termini che appaiono nel linguaggio di Petar I e nei vernacoli della costa montenegrina sono per lo più quelli legati alla sfera della vita quotidiana: avizati (it. avvisare) - informare, avvertire, mettere al corrente, banda (it. banda) - lato, faculet (it. fazzoletto) - quadro di tessuto leggero usato per asciugarsi il sudore e sim., intrada (it. entrata - spazio attraverso cui si entra, reddito) - qui reddito, raccolta; kastig (it. castigo) - punizione, medig (ven. medego) - medico, mentovati (it. mentovare) - menzionare, nominare, spenžati (it. spendere) - sborsare, školj (it. scoglio) - roccia che affiora dalle acque del mare, isolotto. Una menzione particolare la meritano i termini che fanno parte sia del 
vocabolario di Petar I che delle parlate popolari delle popolazioni costiere, ma con più o meno spiccate differenze di significato. Ad esempio, il nome kordun (it. cordone) - viene usato da Petar I nel significato di serie di postazioni militari allineate a scopo difensivo, mentre nei due dizionari delle parlate popolari di cui ci siamo serviti la stessa parola significa "corda che viene cucita su un capo di abbigliamento come ornamento; corda molto grossa". II sostantivo kompanija (it. compagnia) nel linguaggio di Petar I significa "reparto di truppe", che è uno dei significati originali di questa parola, mentre Vesna Radulović cita lo stesso termine nel significato di "comitiva", anch'esso presente nella lingua d'origine. II sostantivo kavalijer (it. cavaliere) è utilizzato da Petar I con il significato di "chi è insignito di un'onorificenza", mentre lo stesso termine nei vocabolari delle parlate popolari significa "seguace, amante". Quest'ultimo significato non viene menzionato nei dizionari della lingua italiana che abbiamo consultato. II nome oficijal (it. ufficiale) nel linguaggio di Petar I ha il significato di "ufficiale militare", mentre lo stesso sostantivo nel dizionario di Vesna Radulović significa "funzionario del tribunale". II significato originale della parola è "militare graduato, funzionario". II termine mortir (it. mortaio, ven. morter) viene usato da Petar I nel significato di "pezzo di artiglieria", mentre nelle parlate popolari lo stesso nome è usato nel significato di "recipiente usato per ridurre in frammenti o polvere sostanze varie". Nel linguaggio di Petar II il sostantivo ponat (it. punto) ha il significato di "segno di interpunzione", mentre nel parlato della nostra costa significa "il tratto di filo che passa attraverso due forellini fatti dall'ago nella stoffa; unità usata per esprimere vantaggio nei giochi".

\section{Italianismi negli scritti di Petar II Petrović Njegoš}

Nel corpus esaminato che comprende gli scritti di Njegoš sono stati registrati 235 italianismi. Di questi termini 110 (46,81\%) compaiono anche nei vocabolari di Musić e della Radulović, 16 parole $(6,81 \%)$ sono state registrate solo da Musić, mentre 20 vocaboli $(8,51 \%)$ sono stati riportati soltanto dalla Radulović. I rimanenti 89 termini $(37,87 \%)$ non sono stati registrati né nel parlato delle Bocche di Cattaro, né in quello di Budua e Paštrovići. Tra le parole non registrate nelle parlate popolari prevalgono toponimi e idronimi, nonché gli aggettivi possessivi da essi derivati. Nessuna delle 16 parole registrate nel corpus appartenenti a queste categorie compare nei vocabolari di Musić e della Radulović. Njegoš usa spesso nomi geografici originali (Trijest, Frijul, Vezuvij), anche se nella lingua montenegrina esistono forme corrispondenti (Trst, Furlanija, Vezuv); inoltre, utilizza anche 
toponimi italiani di alcune località che all'epoca non erano sotto il dominio di stati esistenti sulla penisola italiana - Raguza (it. Ragusa) vecchio nome della città di Dubrovnik; Antifar (it. Antivari) - Bar; skutarski (it. Scutari) - della città di Scutari. Abbiamo registrato anche la forma Boka di Kataro che è stata coniata combinando due toponimi - montenegrino e italiano. La forma Lizonco è molto interessante, perché Njegoš utilizza non soltanto il nome geografico originale (Isonzo), ma anche l'articolo determinativo ad esso collegato, visto che questi due elementi, nella produzione orale, vengono percepiti come una sola parola.

Nella seconda categoria di nomi non registrati da Musić e dalla Radulović rientrano i termini che indicano diverse istituzioni e cariche amministrative. Questo è comprensibile, se si prende in considerazione il fatto che la maggior parte di queste istituzioni era già scomparsa nel periodo in cui i due studiosi hanno effettuato le ricerche. Musić e la Radulović hanno registrato soltanto termini quali providur (it. provveditore, ven. proveditor) - funzionario della Reppublica di Venezia e prokaradur (it. procuratore, ven. procurador)-procuratore legale, pubblico ministero, mentre Njegoš usa anche i termini come bailo (it. bailo) - chi ricopriva la carica di ambasciatore o console nelle colonie venete o fiorentine nel Levante; sindik (it. sindaco) - capo di amministrazione di un comune; princip (it. principe) - chi è investito del titolo nobiliare, doge di Venezia; konte (it. conte) - titolo nobiliare.

Njegoš utilizza anche diversi termini appartenenti al linguaggio dell'economia e del commercio, tra i quali qui citiamo: interes (it. interesse) - somma dovuta da un debitore per la concessione di un credito; ipotekati (it. ipotecare) - gravare di ipoteca; sensal (it. sensale) - intermediario nella compravendita; vidimirati (it. vidimare) attestare l'autenticità con atto pubblico. In questa categoria possono rientrare anche i termini che indicano alcune valute oggi fuori corso: rušpa (it. ruspa) - antica moneta veneziana; cekin imperijale (it. zecchino imperiale) - zecchino reale; luid (it. luigi) - moneta aurea francese; talijer (it. tallero) - grossa moneta d'argento.

Nel corpus esaminato abbiamo registrato anche altri termini, quali cirk (it. circo) - anfiteatro romano; foro (it. foro) - centro dell'antica città romana; koloseo (it. Colosseo) - ampia costruzione aperta impiegata per spettacoli o giochi gladiatori. Queste parole non sono state registrate nelle parlate popolari, ma si può supporre che Njegoš le abbia apprese durante i suoi viaggi alla scoperta delle ricchezze del patrimonio culturale italiano.

La seconda parte del corpus comprende i termini registrati sia nelle parlate popolari che negli scritti di Njegoš. In questo segmento gli 
elementi che richiedono particolare attenzione sono le parole che, pur avendo la stessa origine, presentano alcune differenze di significato. Nella maggior parte dei casi, le parole registrate da Njegoš hanno un significato più generico e più vicino al significato originale, mentre le stesse parole nel parlato assumono un significato più preciso e più stretto. A testimonianza di quanto detto, riportiamo alcuni esempi: nel vocabolario di Njegoš la parola patron (it. padrone) significa soltanto "proprietario", mentre nel parlato lo stesso termine assume il significato di "proprietario di una rete da pesca, capo di un gruppo di pescatori". Njegoš utilizza la parola pošt (it. posto) nel significato di "luogo", mentre nella parlata popolare questa parola significa "luogo conosciuto per l'abbondanza di pesce". La parola spenza (it. spesa) nel vocabolario di Njegoš viene usata nel significato originale di "erogazione di denaro al fine di ottenere beni o servizi", mentre nel parlato la stessa parola significa "acquisto in piazza". II termine buleta (it. bolletta) viene usato da Njegoš esclusivamente nel significato di "ricevuta", mentre lo stesso termine nel parlato significa "licenza di vendita del bestiame". Nel caso di termini che presentano più significati diversi, nel parlato, di solito, i termini in oggetto vengono usati nel significato più frequente, mentre nel vocabolario di Njegoš appaiono in un significato diverso, a volte meno comune e più raro. Come esempio, riportiamo il nome skala (it. scala) che, nel parlato, significa "gradinata", mentre Njegoš utilizza questa parola nel significato di "roccia" che è molto più raro. Un esempio interessante riguarda il termine tutela (it. tutela) che nella lingua parlata popolare significa "protezione", mentre da Njegoš viene usato nel significato di "protettrice" che non viene riportato dai dizionari consultati.

Nonostante questi esempi, bisogna dire che la maggior parte degli italianismi, che appaiono sia nel parlato che nel vocabolario di Njegoš, condividono lo stesso significato. Questa parte del corpus può essere suddivisa nelle seguenti categorie: termini che indicano generi alimentari, parole che denotano capi di abbigliamento e, infine, nomi che si riferiscono agli oggetti e agli aspetti della vita quotidiana. In seguito riportiamo alcuni esempi: barka (it. barca) - imbarcazione di piccole dimensioni, galija (it. galea) - nave veloce e leggera, gondula (it. gondola) - imbarcazione tipica della laguna veneta, trabakula (it. trabaccolo) - piccolo veliero, vapor (it. vapore) - piroscafo, barilo (it. barile) - botte, bistijerna (lat. it. cisterna) - serbatoio per la raccolta dell'acqua piovana, depozit (it. deposito) - magazzino, serbatoio per l'acqua, magazin (it. magazzino) - locale adibito al deposito e alla conservazione di merci, beškot (it. biscotto) - cotto due volte, piccolo dolce cotto a lungo nel forno, cukar (it. zucchero) - sostanza usata per 
dolcificare cibi o bevande, frut (it. frutto) - prodotto delle piante derivante dal fiore, kastradina (ven. castradina) - carne affumicata di agnello, skoranca (ven. scoranza) - pesce affumicato, kamara (it. camera, ven, camara) - stanza, kaseta (it. cassetta) - baule, lama (it. arh. lama) - recipiente di metallo, lincul (it. lenzuolo) - telo che si stende sul tetto, pirun (ven. piron) - forchetta, saket (it. sacchetto) piccolo sacco di carta usato per contenere e trasportare oggetti, dondo (it. arh. donno) - zio, familja (it. famiglia) - insieme di persone unite da parentela, medik (it. medico) - dottore, portantina (it. portantina) barella, pijaca (it. piazza) - trg, riva (it. riva) - obala, školj (it. scoglio) greben u moru, malo ostrvo, butiga (it. bottega) - negozio, buslo (it. bussola) - strumento di orientamento in grado di indicare la posizione dei punti cardinali, ura (it. ora) - unità di tempo pari alla ventiquattresima parte del giorno solare, maškara (it. maschera, ven. mascara) - persona mascherata, multa (it. multa) - pena pecuniaria prevista per determinati reati, miritati (it. meritare) - essere in condizione di poter aspirare a una ricompensa, un favore o un aiuto, resto (it. resto) - ciò che rimane di un tutto o di un insieme, faculet (it. fazzoletto) - quadrato di tessuto leggero usato per soffiarsi il naso, per asciugarsi il sudore e sim.

\section{Livello di adattamento dei prestiti}

Per quanto riguarda il livello di adattamento dei prestiti registrati nel corpus, si può notare che essi appartengono alla categoria dei cosiddetti prestiti integrati. Analizzando il livello di adattamento dei prestiti, Musić parte dalla loro classificazione in base al grado di adattamento a livello fonemico e morfemico. A livello fonemico si possono distinguere tre categorie di parole - prestiti non assimilati, prestiti parzialmente assimilati e prestiti completamente assimilati. Anche a livello morfemico possono essere individuate tre categorie di prestiti, ossia - prestiti non adattati, prestiti parzialmente adattati e calchi semantici. Secondo Musić, il parlato delle Bocche di Cattaro ha la capacità di assimilare con facilità tutti i prestiti provenienti dalle lingue romanze, in particolar modo dall'italiano e dal dialetto veneziano, grazie alle spiccate somiglianze tra i loro sistemi vocalici (Musić 1972, 74). Parlando del grado di adattamento dei prestiti a livello morfemico, Musić parte dalla definizione di Ivan Klajn secondo cui l'assimilazione morfologica consiste nell'aggiunta di desinenze flessionali (quando una parola passa da una lingua analitica ad una lingua sintetica). In caso contrario, cioè, se la parola passa da una lingua sintetica ad una analitica, di tutte le forme flessionali ne viene conservata soltanto una. Musić afferma che la maggior parte degli italianismi nel parlato della 
parte nordoccidentale delle Bocche di Cattaro si sia adattata anche a livello morfemico. Tra i cambiamenti che subiscono questi prestiti il più frequente è il cambio di genere dei nomi, a volte dovuto a diverse analogie, ma molto più spesso al fatto che nella lingua italiana, a differenza dal montenegrino, non esiste il genere neutro. Una parte dei nomi conserva suffissi italiani, mentre alcuni adottano suffissi caratteristici della lingua montenegrina. Alcuni verbi conservano $i$ prefissi originali, mentre altri, subendo l'influenza del montenegrino, assumono i suoi prefissi. Gli aggettivi di solito adottano i suffissi caratteristici della lingua montenegrina, ad eccezione di una piccola parte che mantiene la forma originale.

Le osservazioni precedenti possono essere applicate anche al nostro corpus - i prestiti registrati sono adattati alla lingua montenegrina secondo le stesse regole che valgono per i termini che sono entrati nelle parlate popolari. Questa conclusione vale non soltanto per i vocaboli registrati nel parlato, ma anche per quelli che compaiono esclusivamente nel patrimonio scritto di Petar I e Petar II.

\section{Conclusione}

In questo contributo si è cercato di affrontare la questione della presenza della componente lessicale italiana in tutto il patrimonio scritto dei vescovi e scrittori montenegrini Petar I Petrović e Petar II Petrović Njegoš. La scelta di questo argomento è stata determinata dalla precedente disamina della letteratura esistente dedicata ai due, dalla quale è risultato che quest'ambito problematico non è stato ancora sufficientemente esaminato. I risultati di questa ricerca dimostrano che la componente lessicale proveniente dall'italiano è presente negli scritti di entrambi gli autori, ma che si possono evidenziare alcune più o meno spiccate differenze tra i due. II numero e la varietà di parole di origine italiana nelle opere di Petar I sono relativamente scarsi, specialmente nella sua opera più significativa Poslanice. Gli italianismi sono presenti in misura leggermente maggiore nelle sue lettere, mentre un numero molto esiguo di parole di origine italiana è stato registrato nelle opere poetiche. Per quanto riguarda Petar II, le parole di origine italiana sono presenti in misura assai più rilevante nelle sue lettere rispetto alle sue opere letterarie. Questo potrebbe essere dovuto al fatto che, come già mostrato, Njegoš conosceva la lingua italiana e che aveva frequenti contatti diretti con le aree in cui questa lingua era ampiamente presente in quell'epoca. Infine, va notato che entrambi gli autori hanno tratto una parte del materiale lessicale italiano dai vernacolari della loro terra natale, in cui la componente lessicale italiana si è introdotta relativamente presto a 
causa dei costanti contatti con le zone costiere dell'odierno Montenegro.

\section{Letteratura:}

Andrijašević, Živko. Istorija Crne Gore u 55 priča. Podgorica: Zavod za udžbenike i nastavna sredstva, 2009.

Banašević, Nikola. „Njegoševo učenje stranih jezika.“ Zapisi, III, knj. V, sv. 4, (1929): 193-202.

Boerio, Giuseppe. Dizionario del dialetto veneziano. Firenze: Giunti Editore, 1998. (prvo izdanje: Giovanni Cecchini Editore, Venezia 1856.)

Ćupić, Drago. Lingvistički ogledi o Vuku i Njegošu. Beograd: Naučna knjiga, 1988.

De Mauro, Tullio. Il dizionario della lingua italiana per il terzo millennio. Torino: Paravia, 2000.

Kilibarda, Vesna. Njegoš i Venecija, Venecija i slovenske književnosti. Beograd: SlovoSlavia, 2011. 289-302.

----------„Njegoševo poznavanje italijanskoga jezika.“ Lingua montenegrina, br. 11, (2013): 197-207.

Njegoš i Italija. Podgorica: Institut za crnogorski jezik i književnost, 2014.

Klajn, Ivan. „Strana reč - šta je to?“ Zbornik za filologiju i lingvistiku, br. 10. (1967): $7-24$.

Lipovac Radulović, Vesna. Romanizmi u Crnoj Gori - Budva i Paštrovići. Novi Sad: Matica srpska, 1997.

Romanizmi u Crnoj Gori - Jugoistočni dio Boke Kotorske. Novi Sad: Matica srpska, 2004.

--------- Romanismi lessicali in Montenegro. Budua e Pastrovici. La parte sud-orientale delle Bocche di Cattaro. Roma: Il Calamo, 2009.

Musić, Srđan. Romanizmi u severo-zapadnoj Boki Kotorskoj. Beograd: Filološki fakultet Univerziteta u Beogradu, 1972.

Ostojić, Branislav. Jezik Petra I Petrovića. Titograd: CANU, 1976.

---------O crnogorskom književnojezičkom izrazu. Nikšić: NIO Univerzitetska riječ, 1985.

Stevanović, Mihailo. „Neke osobine Njegoševa jezika.“ Južnoslovenski filolog, knjiga XIX, (1951): 17-31.

Stijović, Svetozar. Slavenizmi u Njegoševim pesničkim delima. Sremski Karlovci - Novi Sad: Izdavačka knjžarnica Zorana Stojanovića, 1992. 
Vukmanović, Savo. (1982). „Petar II Petrović Njegoš u Herceg Novom.“ Boka - Zbornik radova iz nauke, kulture i umjetnosti, br.13-14, (1982): 338.

Vušović, Danilo. Prilozi proučavanju Njegoševa jezika, Beograd: Grafički umetnički zavod „Planeta“, 1930.

Zingarelli, Nicola. Vocabolario della lingua italiana, XII edizione, Bologna: Zanichelli, 2002.

\section{ITALIJANIZMI IZ SVAKODNEVNOG GOVORA U PISANOJ ZAOSTAVŠTINI PETRA I I Petra II Petrovića NJegoša}

$\mathrm{U}$ ovom radu analizira se prisustvo italijanizama koji pripadaju sferi svakodnevnog govora u pisanoj zaostavštini crnogorskih vladika i pisaca Petra I i Petra II Petrovića Njegoša. Ako se uzmu u obzir tvrdnje stručnjaka da posuđenice koje vode porijeklo iz italijanskog jezika ili njegovih dijalekata čine sastavni dio crnogorskih narodnih govora njihovog vremena, kao i činjenica da su obojica odabranih autora bili u prilici da se na različite načine upoznaju sa italijanskim jezikom, čini se da je opravdano pretpostaviti da u njihovoj pisanoj zaostavštini mora biti elemenata italijanske leksike. Ova je tema odabrana i zbog toga što se, nakon analize postojeće literature posvećene pisanoj zaostavštini Petra I i Petra II, došlo do zaključka da se do sada niko nije upustio u detaljnu analizu prisustva italijanske leksičke komponente u njihovim djelima, iako je bilo sporadičnih i usputnih osvrta na postojanje leksičkog materijala venecijanskog ili italijanskog porijekla u jeziku dvojice pomenutih autora.

Ključne riječi: Petar I Petrović, Petar II Petrović Njegoš, italijanizmi, narodni govori, venecijanski dijalekat 